\title{
FUTURE VISION OF GLOBALLY HARMONIZED NATIONAL AIRSPACE SYSTEM WITH CONCEPTS OF OPERATIONS BEYOND YEAR 2020
}

\author{
Satish C. Mohleji and Paul A. Ostwald \\ Center for Advanced Aviation System Development (CAASD) \\ The MITRE Corporation, McLean, Virginia, USA
}

\begin{abstract}
As laid out in the National Airspace System (NAS) Operational Evolution Plan (OEP)_[1], the Federal Aviation Administration (FAA) and the aviation community are planning to make significant investment in NAS improvements over the next ten years. These enhancements involve implementation of Communication, Navigation and Surveillance (CNS) technologies, and automation of ground systems in order to improve efficiency, safety, capacity and security. However, the mode of operations during this time frame is expected to continue as it is today. A number of research efforts are underway to consider significant changes to NAS operations beyond the OEP. This paper presents a vision of future NAS and operational concepts for year 2020 and beyond based on a number of new paradigms that will provide globally harmonized service to conventional aircraft, uninhabited and space-launch vehicles. The key paradigms are: 1) a multi-faceted airport structure of well connected hubs, spoke, satellite and smaller airports; 2) multi-level CNS architecture for seamless and secured operations; 3 ) problem-free flight planning independent of look ahead times; 4) flexible sector boundaries based on equitable workload; and 5) a universal information service assuring uniformity and security of real time information to all stakeholders and service provider Decision Support Systems_(DSS). Potential make up of future air traffic is discussed including a significant number of non-scheduled flights such as on-demand service, charter, travel club, fractional and short-haul intra-city operations. A concept of System Wide Information Management (SWIM) that provides a virtual electronic collaboration space is described. The operational concepts fully support self-delivery and self-separation for appropriately equipped aircraft. New roles for service providers at National, Regional and Local Air Traffic Management (ATM) facilities are defined. A set of
\end{abstract}

performance goals are established considering the impact of several key operational factors. The operational concepts presented are intended to provide accurate real time gate to gate information, and multiple flight options between departure and destination airports to the users.

\section{Introduction}

The air transportation system is and will continue to be an essential component of the world economy, with continued long-term growth in the need to transport people for business and leisure purposes. The global movement towards just-intime production inventories and E-commerce also will significantly increase the importance of aircargo operations. Cargo and passenger demands will create the need for air transportation operators to serve new markets and new airports including many of the 200 airports within 50 miles of a major airport in the U.S. capable of serving small and medium size jets. As the metropolitan areas continue to grow, these airports will provide proximity to air travel for more communities, and will offer passengers more options to fly direct between suburban airports. Customers will seek more flexibility and options tailored to their specific needs increasing the demand for air taxi, charter, fractional ownership, and use of on-demand small low-cost jet aircraft. To maintain a world class service, the future air transportation system must provide the flying public alternatives based on lowest travel costs, suitable schedules, and easy access to the desired airports. Figure 1 illustrates some of these alternatives envisioned to be available. It also must provide aircraft operators the ability to fly anywhere at any time. The most important challenge for the future ATM system will be to maintain and strengthen safeguards that protect the security of passengers, operating personnel, and continuity of system operations. 


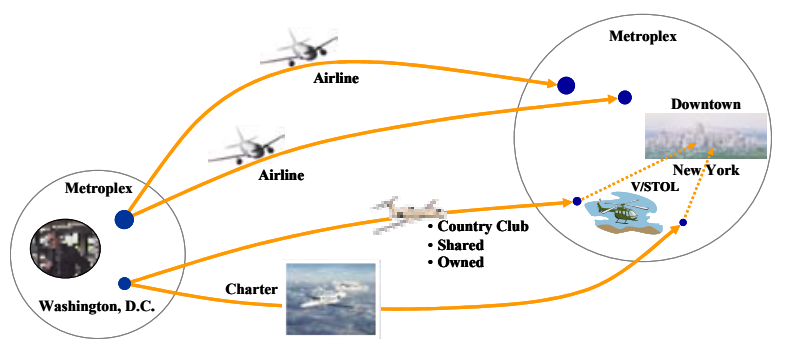

Figure 1. Options for Travelers

Recently, a number of studies [2-16] have presented ideas to potentially revolutionize the future air transportation systems in the U.S. and in Europe. This paper expands upon some of these ideas and adds some new ones to develop wellstructured operational concepts for the year 2020 and beyond time frame. The paper also clearly establishes roles and responsibilities for service providers in various operational domains as well as realistic performance goals based on the results of operational analyses.

\section{NAS Future Vision Overview and New Paradigms}

Figure 2 illustrates major elements of the future global, interoperable and harmonized NAS.
The future vision assumes an expanded role of current CNS/ATM systems to not only cover global flight operations, but also provide service to a wide range of emerging aerospace vehicles including Reusable Launch Vehicles (RLVs), Uninhabited Aerial Vehicles (UAVs), subsonic and supersonic aircraft, and Vertical Short Take Off and Landing (VSTOL) aircraft. The overall objective is to move all categories of users anywhere, anytime, on time, safely, efficiently and economically without adversely impacting the environment.

The existing systems used for CNS services needed for ATM, are located on the ground. Because of the propagation characteristics of the radio-frequency spectrum used for international air traffic control, these ground-based systems cannot cover the entire airspace. This is because of the need for an unobstructed line-of-sight between a surveillance sensor, navigational aid, or communications outlet and the aircraft. The satellite-based CNS technologies can provide global coverage. For aviation security, the future CNS systems will be secured against interference, jamming and spoofing. They would be able to substantiate voice and data communications, provide back ups against system failures and surveillance of uncooperative targets.

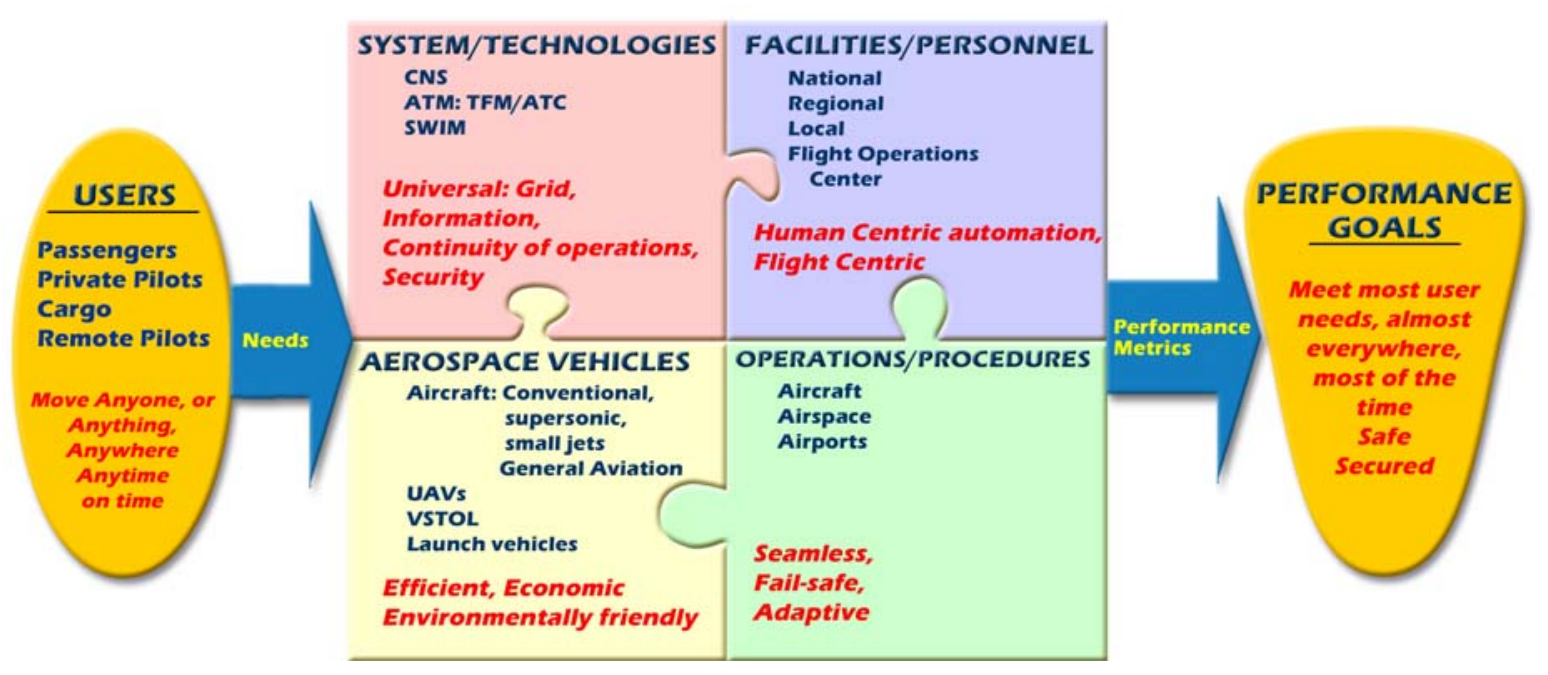

Figure 2. Globally Harmonized NAS Overview 
In order to assure seamless and continuous operations, there is a need to develop a multi-level CNS system architecture incorporating both satellite and ground technologies with standardized data formats, transfer protocols, and universal grids to assure secured capabilities. Multiple Input Multiple Output (MIMO) technology is considered to offer alternatives to SATCOM over land to extend VHF bandwidth for datalinks. For secured and real time information availability, the SWIM will provide a virtual collaboration space for effective information display to all stakeholders. All flight operators will be able to interact with SWIM either directly or via a virtual Flight Operations Center (FOC).

The future ATM system will provide humancentric automation for not only traffic management around weather cells, but also for effective traffic congestion management for most flights from gate to gate by minimizing prediction uncertainties.

Because of seamless surveillance that is not dependent on the radar boundaries, the functions of Air Route Control Centers (ARTCCs) and Terminal Radar Control (TRACON) facilities will be assumed by a common Regional facility. The functions of the current regional Traffic Management Coordinators (TMC) and the D controllers will be taken over by a Strategic Controller (SC). The roles of the National Facility at the Air Traffic Control System Command Center (ATCSCC) and the local towers will continue to remain largely as they are to today.

In order to permit most users to fly anywhere at any time, the future airspace design will support 4D navigation flight profiles in combination with non-4D aircraft. Most profiles will be flight-centric, i.e., permit each user to customize their own flight plan. These profiles will be like a tunnel in space separated from other flight profiles. Wherever intersections are unavoidable, each aircraft will be permitted to arrive at the intersection within a time window consistent with its performance within the available speed envelope over every desired flight segment. Moreover for efficiency, the airspace design will provide the flexibility for the pilots to directly interact with the ATM system to change flight paths if weather/wind conditions affect the negotiated 4D contract. To deal with complex and dynamically changing requirements for the use of airspace, the service providers will have accurate information and decision support tools to make timely decisions for problem-free operations. The problem-free flight planning will be based on the aircraft self-delivery tolerance at each point of conflict that defines the length of a conflict protection volume. The breadth and height of the volume will respectively be based on the Required Navigation Performance (RNP) and the Required Vertical Separation Minimum (RVSM). Since the size of the aircraft protection volume against potential conflicts will be constant, it will not depend upon the look ahead time. The goals of the future NAS are to meet most user requirements of desired flight profiles including times without compromising safety.

\section{Future Demand: Drivers for Change}

It is difficult to precisely predict the air traffic demand that would exist in 2020 timeframe. This is because numerous factors contribute towards determining where and how many people will need to travel by air, and what kind of services will be available to meet their needs. However, technology and industry trends coupled with other predictions can help establish what qualitative changes to traffic flows can be expected. In turn, these expected changes could help determine the drivers that will shape the needs for the 2020 ATM system.

There are a number of new aircraft types expected in the next 20 years that might have an impact on air travel. These include UAVs, RLVs, and civil tiltrotors. By 2020, this paper assumes that the UAVs will not be carrying people, but on a regular basis will be used for security and surveillance. UAV cargo operations are a remote possibility. Although procedures to accommodate UAVs, RLVs and VSTOLs will need to be established, these aircraft are not likely to have a huge impact on the overall future demand, and thus are not drivers for shaping future ATM concepts. However, the new aircraft types that will likely to have the largest impact will be the inexpensive jets. While predictions vary, there is little doubt that these aircraft have the potential to greatly reduce the fixed and variable costs of private jet operations, thus expanding their appeal. Several commercial companies are in the process of developing small jets for certification that will have 
significantly reduced operating costs. Predictions regarding market penetration and total market size vary. There are other aircraft also under development that are likely to have some impact on future demand. These include the Farnborough F1, a turbo prop that is expected to cruise at jet altitudes (at 325 knots) and land on very short $(<2000 \mathrm{ft})$ runways.

Not just the aircraft will be changing in the future, but also the airline industry will be going through a number of changes. Because of economic factors and threats from the low cost carriers, the large network carriers are altering their business and operational models to both increase their service offerings and reduce costs. New alliances are likely to emerge as well as some major airline merges could be expected. New business models are emerging that will likely increase the flight options offered to the travelers. One such business model is fractional ownership. The fractional ownership model originally arose as an option to corporate or individual ownership of private aircraft. Rather than buying or leasing an entire aircraft, participants in fractional ownership programs purchase a share of an aircraft and pay a fixed monthly management fee. These participants are then allotted a given number of flight hours based on their share size. The travelers enjoy the flexibility that comes with corporate ownership, but at a significantly reduced initial and recurring cost. Also recently, ideas were floated to establish travel clubs where passengers fly on flights restricted to members-only potentially reducing the hassle-factor of air travel. In future with the growth of such unscheduled operations, the ATM system will have to deal with a much higher percentage of unscheduled demand.

\section{Future Traffic Flows}

The above trends in demand are likely to contribute to major changes in air traffic flows. It is expected that the following transformations will emerge by the year 2020 .

- Increased Unscheduled Traffic Demand: Due to the increases in fractional ownership, availability of low-cost private jets, the growth of low-cost point-to-point carriers, and the operating shifts expected for the network carriers, there will be a major change in the overall traffic demand resulting from increased unscheduled on-demand operations.

- Increase Usage of Satellite Airports in Metropolitan Areas: The increase in the number of aircraft privately operated either as corporate, fractional, or charter will tend to increase the number of flights to satellite airports in or near major metropolitan areas. These flights will tend to focus on the final destination of the traveler (e.g., airport closest to the business meeting, vacation home, etc.). Because of their smaller size, these flights are also able to use smaller airports rather than compete for the large commercial airports. Since the future CNS technologies will enable precision approaches to almost all runways, these airports will be able to provide service in most weather conditions.

- More Constant Demand: As more and more network carriers de-peak their hub schedules to remain competitive with low-cost carriers, there will be fewer demand peaks driven by the airline banking, which presently occurs due to the hub and spoke system of operations. As the metroplex areas grow, the future terminal areas will include services to satellite airports around the hubs resulting in a shift in demand. While there still will be busy times due to passenger desires, the traffic peaks and valleys due to banks of arrivals and departures at major hubs will likely decrease, while smaller jets may operate out of satellite airports.

- Increase Uncertainty in Daily Traffic Flows: Today, the traffic contributing to most of the congestion largely consists of flights from the scheduled air carrier operations. However, due to significant increase in non-scheduled operations of private and fractional ownership together with changes in airline business models (that enable them to be more responsive to changing passenger demand), the daily traffic flows will become increasingly more difficult to predict.

\section{Airport Structure to Enhance Future Capacity}

Today most of the 30 airports operating as hubs have demand exceeding capacity during 
several hours in a day. This results in extensive delays that get worse during bad weather. As a part of the OEP, the FAA has planned to build 12 new runways at major airports. With these runway additions and the planned OEP enhancements, the capacity at most hub and spoke airports will increase. However, in order to deal with growth in traffic by 2020, additional airport capacity will be required to keep the delays to a minimum.

As the metropolitan areas continue to grow with the population as well as business centers moving out into the suburbs extending 50 miles or beyond, there are many satellite airports existing today that not only could provide proximity to several businesses and communities, but also additional capacity to NAS. Direct flights between suburban satellite airports will offer passengers more travel options among high population areas and business centers bye-passing busy airports. In order to provide easy ingress/egress to/from these airports, a multi-modal system may be used, but it involves significant time and funding to develop. In this paper as shown in Figure 3, a multi-facet airport structure is defined that not only links hub/spoke and spoke/spoke airports as today, but also connects satellite airports providing suburbandirect services. In order to provide links between downtown and suburban business centers with satellite and hub/spoke airports, VSTOL or helicopter air-taxi operations are considered.

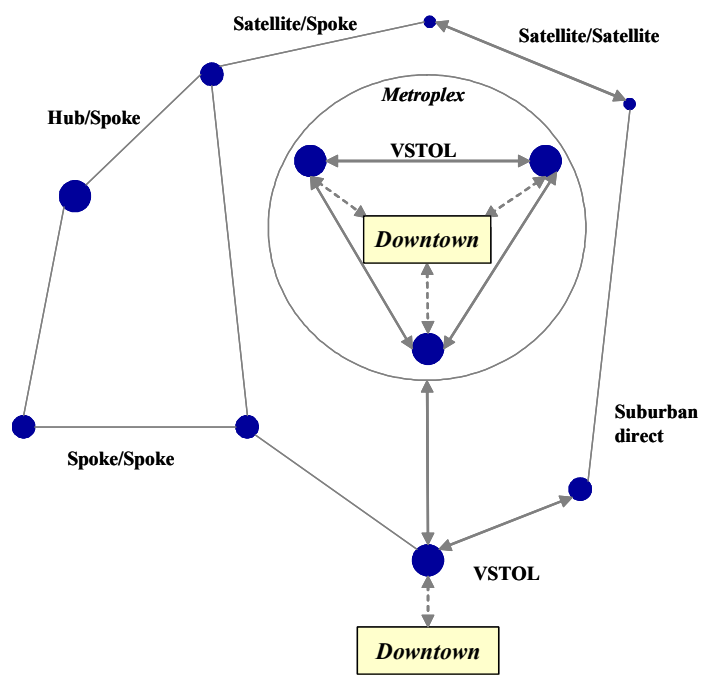

Figure 3. Multi-facet Airport Structure
Currently there are 420 airports in the U.S. with paved lighted runways longer than $5500 \mathrm{ft}$ with a control tower operated by the FAA. These airports can efficiently provide service to Regional and business jets that currently use major hub airports. Out of these 420 airports, there are 126 airports within 50 miles of a major hub. There are an additional 68 airports with the above characteristics with non-FAA towers. These 194 airports are well equipped to accept smaller jets. If these jets operate out of smaller airports, it would result in potentially freeing up some capacity at hub airports. Moreover, these airports could efficiently handle suburban-direct flights increasing flight options for passengers. Figure 4 shows how the existing 30 hubs and above 420 airports match up against various population areas. The population data in the figure are based on the 2000 Census. The tower counts were for 2001 operations[17]. As shown, 172 airports are located near low population densities. But the other 248 airports (in addition 30 hubs) could easily become a part of the future NAS airport structure to enhance capacity. Although some of these airports are already operating as a spoke airport, they need to be interconnected with most other airports in the future.

\section{Multi-level CNS Architecture Concept}

Global CNS services will be provided using a combination of ground based and satellite systems. In order to satisfy continuity of operations, security, safety, and capacity needs, the ground systems are considered to be multi-functional and redundant. For example, the timing for communication system will be used to generate back up navigation capability. The surveillance service will be provided by a combination of radar, secondary surveillance, and aircraft position reports. Figure 5 illustrates a multi-level CNS concept. The key new technologies are the software radio and a surveillance data network. The software radio and internet networking protocols enable global connectivity independent of the underlying physical channel. 


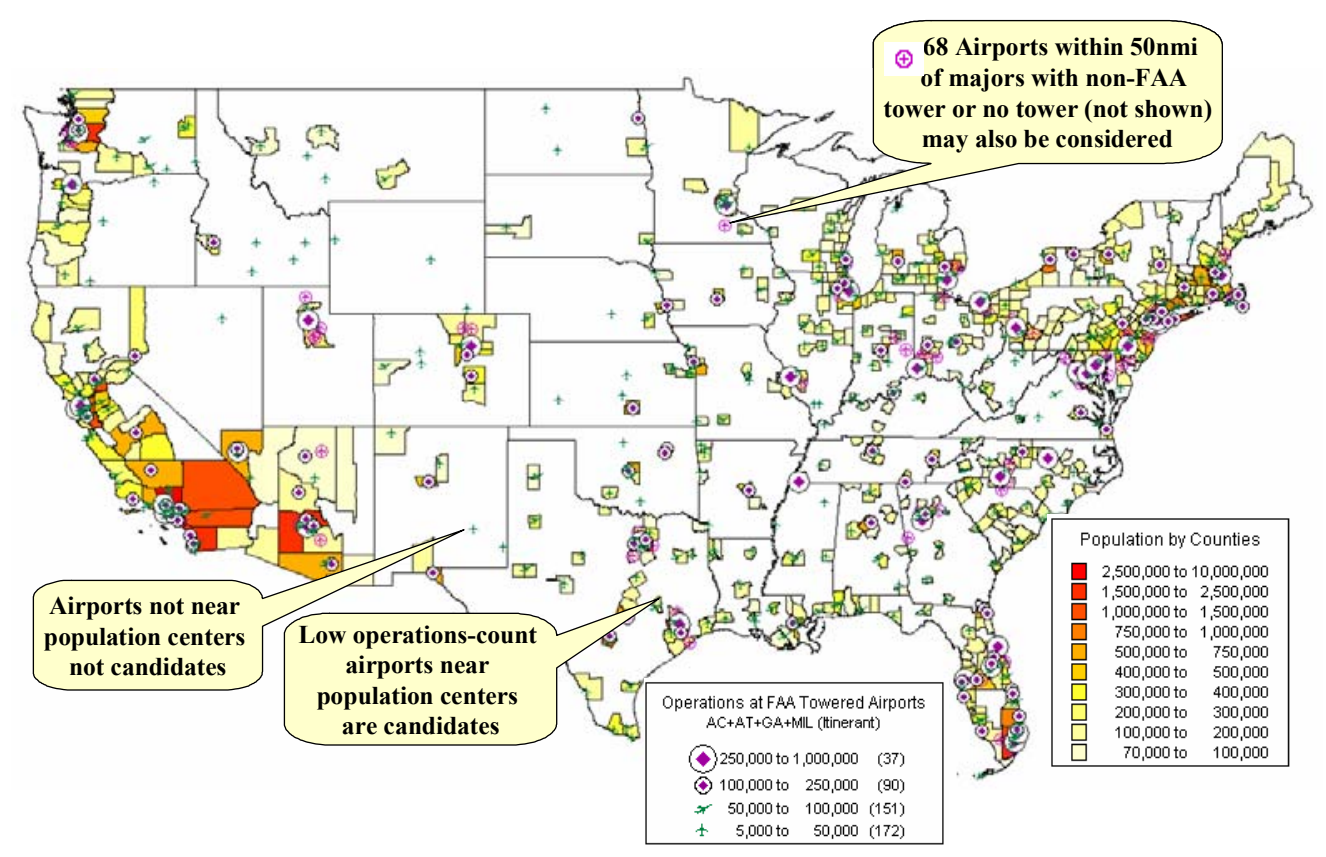

Figure 4. Potential Airports to Enhance Future Capacity

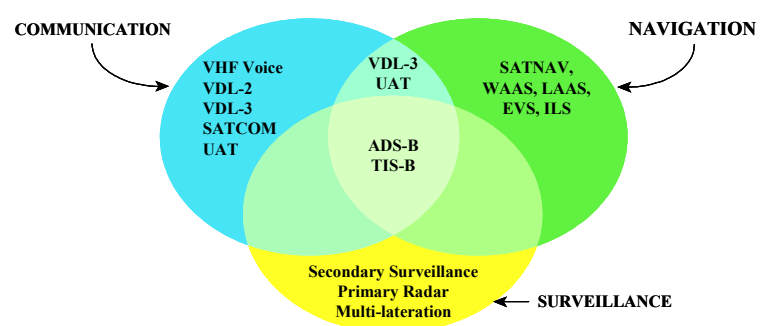

Figure 5. Multi-level CNS Concept

The basic air-ground communication ATM services will be provided via data communications with voice used for real time, critical, non routine communication. The communication architecture is based on a global communications grid to maximize the use of internet technology. The global grid architecture is network-centric (i.e., a common global network connects all users who have addresses on the network), extensible (i.e., modular, reconfigurable, and adaptable to technology insertion), and including layered security (i.e., protection matched to perceived threat). New airground communication services provided include air-air communications and routing, multicasting, access to strategic ATC information, voice over Internet Protocols (IP), and seamless communication. Broadcast services are provided via the Universal Access Transceiver (UAT). This architecture accommodates a range of individual communication systems including Very High Frequency (VHF) Data Link (VDL)-2, VDL-3, and SATCOM via the software radio for users desiring maximum communication capacity and flexibility. Multiple Input Multiple Output (MIMO) technology is used to increase VDL-3 capacity if needed. Users employing VDL-3 or UAT can use the timing signals for a back up navigation service.

The navigation and landing service is predominantly provided by augmented GPS and Galileo SATNAV systems. The augmentations are provided by Ground and Satellite-Based Augmentation Systems (i.e., GBAS and SBAS) to provide differential corrections and integrity monitoring. Users desiring all weather operations will equip with enhanced vision system to "see" on the airport surface and as an integrity check during the landing operation. Users desiring protection against jamming of SATNAV signals will integrate an Inertial Navigation System (INS) with the SATNAV receiver or derive navigation information from a back up network of navaids (or VDL-3 or UAT derived navigation) interconnecting the top 
airports. Instrument Landing Systems (ILS) could be retained at some runways as a precaution against SATNAV jamming during low visibility.

The surveillance service will be provided by "fusing/integrating" data from all available sources (i.e., automatic aircraft reports, secondary surveillance, multi-lateration of UAT and secondary surveillance signals, and primary radar). The architecture employs a distributed client-server network and establishes connectivity via the planned FAA Telecommunication Infrastructure. As a minimum, the following surveillance information is generated on each aircraft: ID, 3D position and velocity (both "tracked" and "untracked"), time, aircraft intent, and Quality of Surveillance (QoS). The QoS accounts for the surveillance sources, geometry, accuracy, and integrity. Authorized users/clients select the appropriate information for air situation display, and specialized processing (e.g., conformance monitoring and alerting). This architecture gives all authorized users (e.g., FAA and NORAD) the same airside picture.

\section{Future ATM System: Flight Planning and Execution}

Current ATM functions include Traffic Flow Management (TFM) that deals with delaying and rerouting flights due to anticipated congestion at airports or bad weather, and Air Traffic Control (ATC) that keeps aircraft separated from other aircraft, terrain or obstacles. The challenge for the future ATM system will be to dynamically manage all traffic congestion while dealing with significant amount of unscheduled demand (i.e., demand continually changing over time and space).

In order to deal with aggregate congestion and weather, the strategic planners at the National facility will periodically assess the capacity of all resources in NAS, and identify periods when the projected demand exceeds capacity of NAS elements (airspace/airports). Since most flight operators will have control over their flight routes, altitudes and times, they will interact with the SWIM to obtain the overall demand/capacity information required to plan their flights. Since a majority of aircraft will potentially be able to fly 4D navigation, their space/time relationship could be precisely established within a small airspace volume, once the flights are predicted to leave the gate with certain level of certainty. This will allow the users to collaborate with the ATM system to establish problem-free flight plans from gate to gate thereby maximizing airspace use.

It is envisioned that this collaborative flight planning will apply to all users, including the schedule airlines, unscheduled operators, various types of general aviation users, and various government users (military and other government agencies). Once the initial flight plans are submitted, the ATM system will determine if there are any predicted problems, and provide the affected users advance notification of options for flight plan changes that solve the predicted problems. Such users will be expected to evaluate these options, select those acceptable to them and revise their plans. Successful implementation of this process will improve the overall estimation of airspace congestion, and provide for NAS resource allocation leading to minimum required delays.

Strategic controllers at a Regional facility will monitor those aircraft assigned to them for problems of a strategic nature. These include situations where flights are not able to follow the agreed plan resulting in one or more flight's trajectory that is not problem-free. The strategic controller may also respond to a situation initiated by a pilot when there is a need to replan the flight's trajectory due to sudden turbulence or aircraft problems but involves no conflict. The strategic controller will monitor the NAS resources assigned to them and will respond to dynamic situations that impact the resource capacity such as severe weather. Also, the strategic controller within a Regional level facility may interact with another strategic controller, within the same facility or within another facility, to coordinate the resolution of the problems that have been identified with one or more flights under the responsibility of the other strategic controller. Consequently, coordination may occur with a strategic controller in another Regional facility when the inter-facility problems occur, as well as coordination may occur with personnel at the National level facility to address predicted regional congestion or other problems that may have an impact beyond the adjacent Regional facilities. 
By dealing with a number of problems at a strategic level, it is envisioned that this concept will increase responsiveness in dealing with airspace complexity and traffic congestion alleviating most of the traffic flow restrictions. The consolidated decision-making process by combining the functions of current TMCs and D controllers will result in reduced decision times. Figure 6 illustrates the concept of interaction among various strategic controllers.

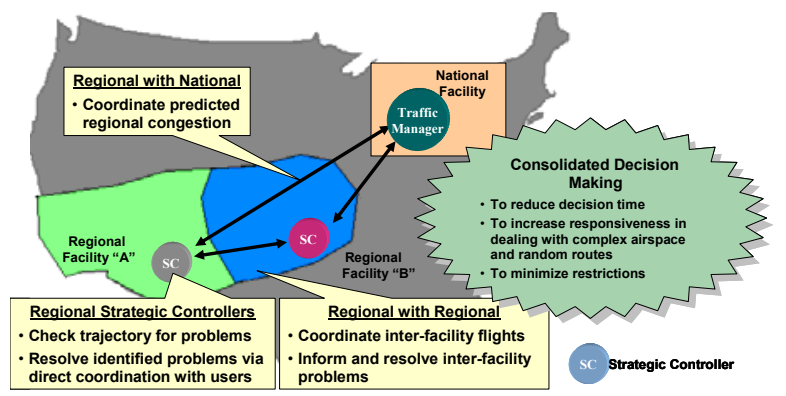

Figure 6. Interaction Among Strategic

In the future ATM system concept, direct interaction could also take place between pilots and Regional level strategic controllers. A pilot may initiate an interaction under conditions when a flight could no longer meet times at waypoints due to stronger than predicted winds, or encounter adverse local weather situations resulting in a need to replan the flight's trajectory. The resulting interaction between the strategic controllers and the pilot will be to negotiate and coordinate the required trajectory changes (changes to the waypoints, altitudes, and desired times of arrival at the waypoints). This interaction will iterate until the strategic controller confirms that the changed trajectory is problem-free, as shown in Figure 7. The strategic controller will ensure that any trajectory changes have been sent to the SWIM for information sharing purposes. At times, depending upon the situation, a third party such as the dispatcher may also be involved in the trajectory negotiation. Strategic controllers may also directly coordinate with non-4D flights to resolve situations such as multi-aircraft encounters.

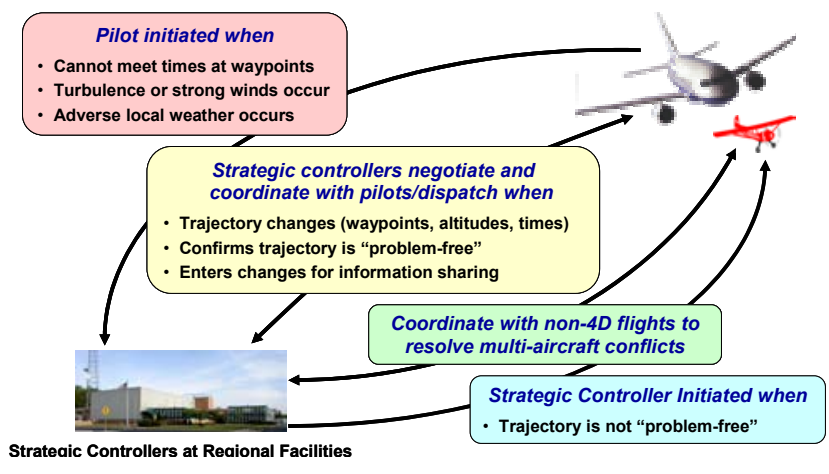

\section{Figure 7. Interaction between Pilots and Strategic Controllers}

The tactical controllers will be primarily responsible for monitoring the aircraft conformance to the negotiated flight plan and addressing any problems that may arise due to the dynamic nature of the situation. While a tactical controller will continue to work a defined volume of airspace, it is envisioned that the definition of this airspace will be much more flexible than in today's environment. Sector boundaries (including vertical floors and ceilings) will be proactively adjusted to establish a volume of airspace for each sector that balances the workload among tactical controllers within a facility. For example, an area of severe weather may be occurring within a sector. Traffic may be rerouted around this area of weather. As a result, an adjacent sector may be predicted to get overloaded due to rerouting of affected flights into that sector. As such, the sector affected by the severe weather will be predicted to have less than the normal amount of traffic due to the aircraft being rerouted out of this sector. Consequently, the boundaries of these sectors are adjusted to provide for flexible utilization of airspace to enhance system efficiency and more equitable distribution of workload among controllers as shown in Figure 8.
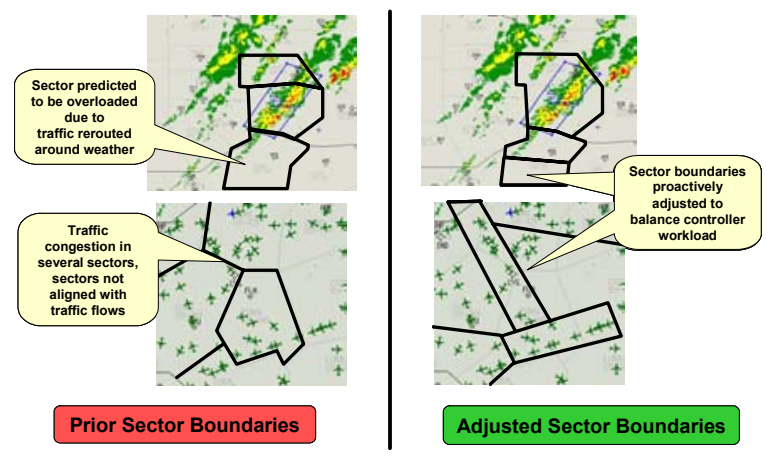

Figure 8. Flexible Airspace Utilization 


\section{Flight Operations: Self-Delivery and Self-Separation}

By 2020, a vast majority of users are envisioned to be capable of flying $4 \mathrm{D}$ navigation. A 4D flight plan contains a trajectory that includes waypoints specified by latitude, longitude, altitude, and the desired time of arrival at each waypoint with a specified time tolerance at each waypoint within which the flight will arrive at that waypoint. These flights will have a problem-free trajectory to avoid weather or traffic congestion that is projected to occur along the way when the flight planning process was done. Each trajectory represents a contract, where the flight will self-deliver at the waypoints and meet the scheduled arrival time at the destination airport within the specified time tolerances using onboard speed adjustments. Once the aircraft is assured to stay within the specified time window, which when translated into a longitudinal distance could help establish the length of a protection volume of airspace at each waypoint. The lateral dimension is defined by RNP and the vertical dimension is based on an altitude deviation of $+/-250 \mathrm{ft}$ (from RVSM), as shown in Figure 9. Based on the aircraft performance within the volume 99 percent of the time, the prediction of the protection volume becomes independent of look ahead time.

As shown in Figure 10, two 4D-equipped flights flying at the same altitude in-trail could maintain self-separation [Note the term selfseparation describes various situations where separation responsibility has been delegated to the aircraft involved in the situation for a defined period of time.] from each other by station keeping. In situations where two 4D-equipped flights approach an unavoidable intersection in a slow closure rate situation, the ground system will permit the aircraft to assume responsibility for selfseparation (Figure 11). However, as shown in Figure 12, the controller will need to monitor 4D aircraft approaching each other at a high closure rate, and could maneuver the second aircraft reaching the intersection to maintain separation. Non-4D users will file flight plans based on the desired routings and altitudes. In such cases, because of times at intersections will have to be predicted by the ground system (and not assured), the ATM system and the controllers will monitor these flights to maintain separation, as shown in Figure 12. Note that under VFR conditions, non-4D flight operations may be permitted to maintain selfseparation. As such, in situations where one flight is $4 \mathrm{D}$-equipped and one flight is non-4D equipped, the controller will attempt to resolve a potential conflict by maneuvering the non-4D equipped aircraft. In situations when both aircraft are non-4D, the controller may maneuver either or both aircraft as needed, as shown in Figure 13.

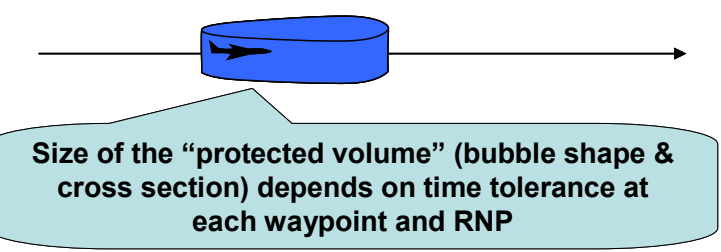

Figure 9. Self-delivery of 4D-equipped Flights

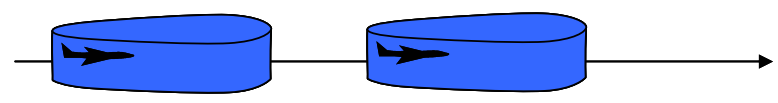

Figure 10. Station-keeping of 4D-equipped Flights

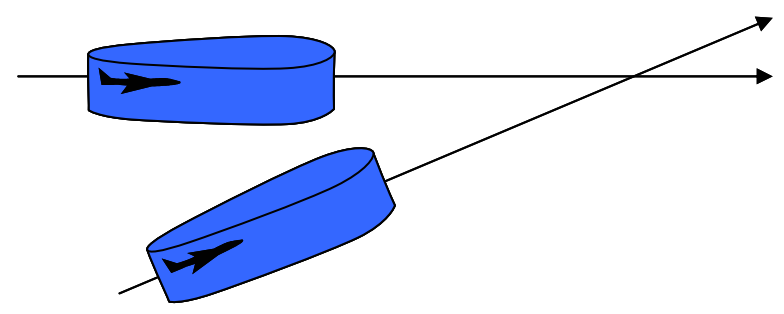

Figure 11. Self-separation of 4D-equipped Flights

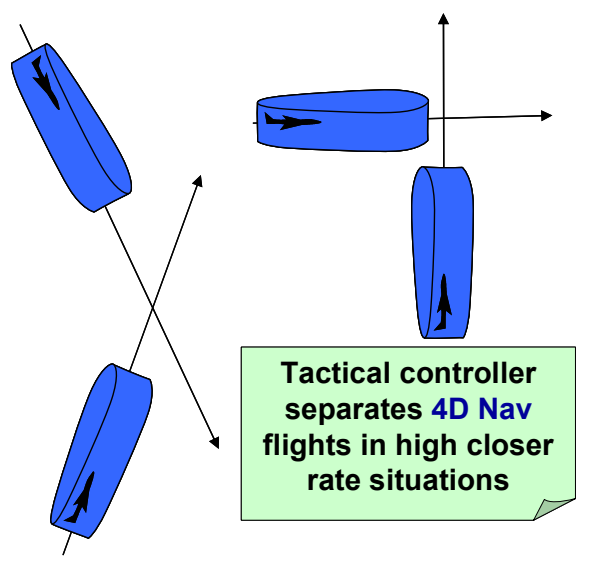

Figure 12. Separation of 4D-equipped Flights in High-closure Rate Situations 


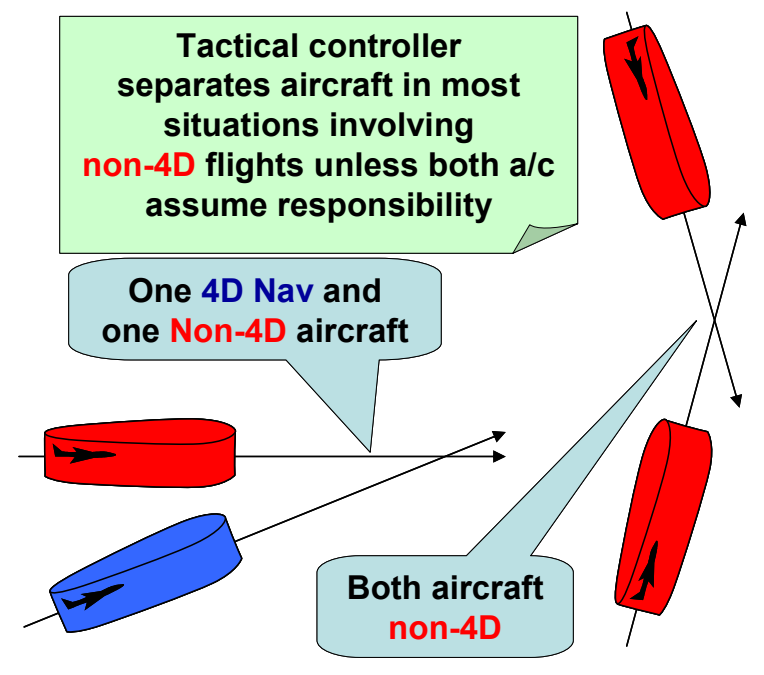

Figure 13. Situations Involving Non-4D Equipped Flights

\section{System Wide Information Management (SWIM)}

The NAS can be viewed to operate at three layers. The uppermost layer is comprised of automation systems that support the users and service providers. These systems are often referred to as Decision Support Systems. Included are also those systems that provide the Human-Computer Interface (HCI). The middle layer is the information layer, as it contains the data and information required by the upper layer. The lowest layer can be referred to as the infrastructure. It is comprised of the hardware and lower-level software components supporting the upper layers, as well as communication mechanisms supporting interaction between the various components that comprise the NAS. Figure 14 shows the primary providers and consumers of information who interact with the System Wide Information Management (SWIM) capability, which is largely resident at the middle layer. The purpose of the SWIM is to manage data and information system-wide across the NAS. As shown, the SWIM will accept information from a wide variety of sources. The SWIM will secure and protect this information and provide the right information to those who need it, when they need it, and wherever they need it. The SWIM also provides a virtual electronic collaboration space that offers easy access to all users and supports various collaborative schemes in real time.

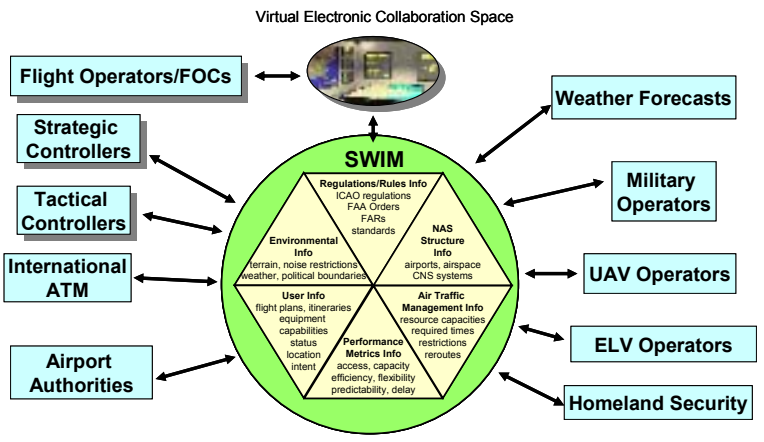

Figure 14. System Wide Information Management

Providing a common, consolidated and consistent operational picture is a primary requirement for SWIM. All of the information and data required by the NAS users and the NAS component systems will be residing in and be managed by SWIM. This consolidation of data and information in one place provides the benefit for each user to establish only one interface with SWIM for exchanging a multitude of data and information.

"Information" is data that is relevant, timely, and actionable. It is relevant if it pertains to the problem at hand. It is timely if it is delivered in a time frame that makes it useful for solving a problem. It is actionable if it forms a basis upon which to decide or act.

As shown in Figure 14, six categories of NAS information managed by the SWIM are identified as follows:

- Regulatory and Rules Information: Includes International Civil Aviation Organization (ICAO) regulations, FAA orders, Federal Aviation Regulations (FARs), and standards

- Environmental Information: Includes information pertaining to terrain, obstacles, landmarks, political boundaries, weather, and noise restrictions

- NAS Structure Information: Includes information pertaining to airports, airspace, and Communication, Navigation and Surveillance systems supporting the NAS

- Flight Operator Information: Includes information pertaining to those who intend to and are operating within the NAS. The 
information includes flight plans, trajectories, equipment, capabilities of the flight, status, location and intent

\section{- Air Traffic Management Information:}

Includes information pertaining to NAS system resource status and capacities, constraints, and other decisions related to required times of arrival and desired routings of flights

- System Performance Metrics Information:

Includes information describing the performance of the NAS in terms of capacity, efficiency/delays, safety, flexibility, predictability and access

A quick scan through the above categories reveals the challenges facing SWIM in managing the information that is very diverse in nature: some is predictive over hours and days; some describe the current state of the NAS; and some information is historic. SWIM must be capable of managing this wide range of information. A NAS-wide plan for information management will be essential to accomplish the goals of SWIM in a timely and efficient manner.

Many of the concepts presented here, such as collaboration and delegation, rely on the concept of "shared situational awareness". The establishment of SWIM will greatly simplify interoperability over the entire NAS and support improved situational awareness.

It is unclear at this time if SWIM is an independent system within the NAS or are capabilities built into other systems. Either way, it is essential that the SWIM capabilities be recognized as mission-critical and developed as such.

There are a number of desired SWIM characteristics. The system should provide a common interface to exchange information regardless of the users' location or type. It should maintain consistent information independent of the location of stored data or processors. Maintaining consistent information also ensures that any copies of data or information are consistent with the original. Some specific characteristics of SWIM are: 1) SWIM must support access to authorized users and prevent access by unauthorized users; 2) SWIM must customize information for each user; 3) SWIM must support continuous operations during software updates, hardware updates, and periods of testing or certification following such updates; and 4) SWIM must continue to provide services during any system failures.

\section{A Scenario to Illustrate Flight Operations}

Figure 15 illustrates some of these concepts as they relate to the operations of different types of flights. It is envisioned that the majority of future users will file a flight plan with a 4D trajectory that will be free of any problems with weather or traffic congestion. However, as the situation changes while the aircraft are airborne, the flight may dynamically replan to deal with the changes. The flight may have constraints imposed, such as an adjustment to the scheduled time of arrival at a waypoint, to solve the problems. These resolutions are coordinated between the flight and the ATM system, and the flight will meet the constraints by following its revised flight plan.

Unequipped non-4D aircraft will file flight plans as today. These flights will specify their intent in terms of routing and altitude but not times (time tolerances) at waypoints. As discussed earlier, the separation responsibility will depend upon the type of operations: for non-4D flights the controller is responsible for separation, while for 4D flights responsibility for separation may be shifted to the pilot depending upon the geometry of the flight paths and aircraft speeds.

Segregation of some segments of airspace is envisioned where different operations or a mix of aircraft performance occur in an otherwise complex or congested area. For example, VSTOL operations into and out of major airports could use dedicated corridors to help reduce the complexity and maintain safety during such operations.

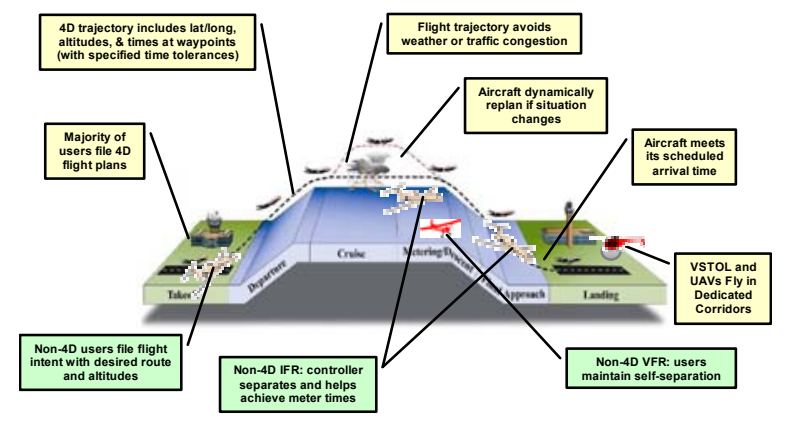

Figure 15. Flight Operations 


\section{Establishment of Future NAS Performance Goals}

Although a number of concepts have been proposed in the literature to revolutionize future ATM systems, in reality, the current NAS will evolve gradually through phases. For successful realization of future NAS vision in this paper, a set of performance goals are established using a rationalized analytical approach. The goals are consistent with the NAS metrics considered in the FAA program development.

\section{Delays (Efficiency)}

Figure 16 shows the causes of delays in current NAS [18]. As shown, the future CNS/ATM systems will be able to potentially alleviate volume and weather, i.e., 69 percent of the delays. However, it may be difficult, though not improbable, to reduce the other delays caused by convective weather, runways not usable and equipment failures.

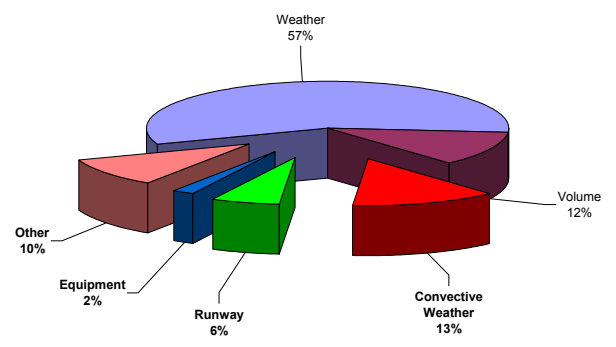

Figure 16. Causes of Delay in NAS

\section{Safety}

Figures 17 and 18 show the causal factors [19, 20] for incidents and accidents during the last ten years. As shown in the figures, the future visionbased concepts are likely to mitigate 96 percent causes of incidents and 72 percent causes of accidents.

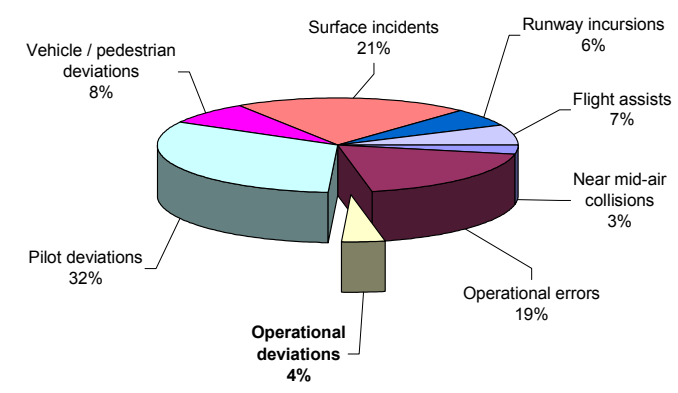

Figure 17. Causal Factors for Incidents

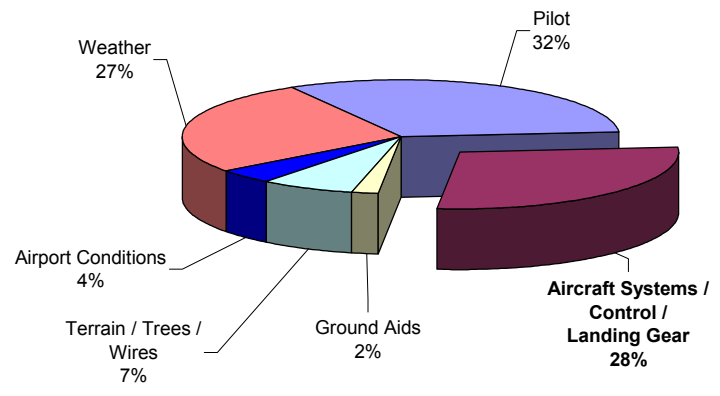

Figure 18. Causal Factors for Accidents

\section{Capacity}

As discussed earlier, developing an infrastructure to fully and effectively utilize 248 airports beyond the 30 hubs[17], will provide adequate airport capacity through 2020 .

\section{Flexibility}

Figure 19 shows causal factors of ATM/airspace restrictions in current NAS[21]. Future concepts will potentially eliminate most of the 97 percent restrictions due to weather, poor visibility and traffic congestion.

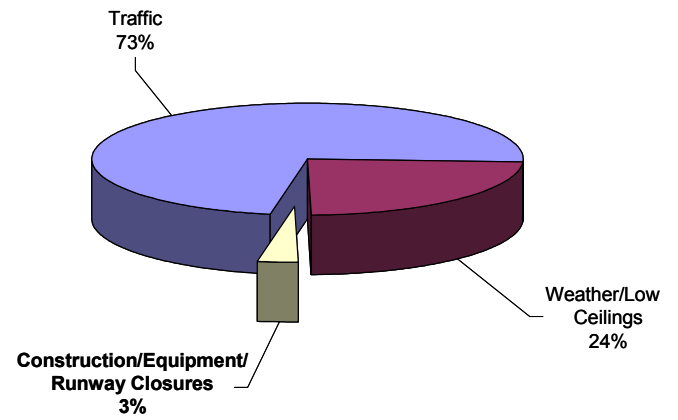

Figure 19. Causal Factors for ATM/Airspace Restrictions

\section{Predictability}

Based on problem-free flight planning with a high percentage of aircraft flying 4D navigation, it is envisioned that only a few aircraft will require flight plan changes.

\section{Access}

Based on the current data[18], instrument meteorological conditions exist 15 percent of the time. Out of these, CAT II/CAT III conditions occur less than 25 percent of the time. As such, the 
future CNS systems will support precision approaches to all runways 96 percent of the time (i.e., up to CAT I weather minima). Most users will be able to fly individual flight profile-based tunnels in airspace.

\section{Workload}

Flexible sector boundaries will be established based on a uniform number of flights simultaneously handled by each controller to equitably distribute workload among most controllers.

\section{Security}

The future CNS/ATM systems will assure continuity and security for all operations at all times.

\section{Summary}

This paper described a vision of CNS/ATM systems for year 2020 and beyond, including a number of operational concepts that consider major shift in paradigms from post-OEP NAS. These concepts maintain the importance of people making decisions and using their best judgment to manage operations. Air/ground automation with accurate information on where the aircraft are and will be, combined with decision tools for managing complex traffic flows, will allow service providers to plan and monitor more traffic in the same amount of airspace, thereby enhancing security, safety, capacity and efficiency. Many of the improvements in safety, efficiency, and capacity will result from the advances in automation, and seamless Communications, Navigation and Surveillance coupled with information sharing to optimize decisions. Broader information availability will be combined with automation that can analyze and predict the impact of changes in traffic levels, routes flown, or weather conditions.

Although discussion of some of the ideas related to the concepts presented in this paper has been going on for some time, this paper attempts to consolidate these ideas along with some new ones into a well coordinated and a structured vision of future NAS. It is intended to provide a realistic perspective of NAS in 2020, and to help establish a roadmap for phased evolution towards the goals established here. Also it is hoped that some of the key ideas would help the aviation community to set a clear direction for research and development of a globally harmonized air transportation system.

\section{Acknowledgements}

The authors thank Mr. T. P. Berry, Mr. A. R. Lacher, Ms. D. E. LaTour, Mr. J. D. Nickum, Mr. F. W. Seelig and Mr. M. J. Zeltser of The MITRE Corporation's Center for Advanced Aviation System Development for their valued discussions and support. The authors are grateful to Ms. M. T. Caldwell and Ms. A. R. Signore for preparing and formatting the final manuscript.

\section{References}

[1] December 2002, National Airspace System Operational Evolution Plan, Version 5.0, Federal Aviation Administration.

[2] 29 October 2002, National Airspace System Concept of Operations and Vision for the Future of Aviation, Update Version 4.5, RTCA.

[3] Runnels, M. H. et al, September 1995, Concept for Future NAS Operations, MITRE Technical Report, MTR95W0000107, Vol. 1, 2, 3.

[4] Kingsbury, J. A. and G. L. Klein, January 1992., Advanced AERA Capabilities, A High Level Description, MITRE Technical Report, MTR91W00158.

[5] Braff, R., January 1975, Self Delivery Terminal Area Control Concept Using MLS, MITRE Technical Report, MTR-6820.

[6] Mohleji, S. C., November 1974, Error Analysis of a Ground-Based Metering and Spacing System Using MLS, MITRE Technical Report, MTR-6796.

[7] Mohleji, S. C., November 1992, Rate Independent Metering (RIM) - Concept for Integrated Separation Assurance and Traffic Flow Planning, Proceedings of the $37^{\text {th }}$ Air Traffic Control Association Annual Meeting, Atlantic City, NJ.

[8] February 2002, The NASA Aeronautics Blueprint - Towards a Bold New Era of Aviation. 
[9] Sietzen, F., August 2002, New Blueprint for NASA Aeronautics, Aerospace America.

[10] June 2001, Air Traffic Management: Revolutionary Concepts that Enable Air Traffic Growth while Cutting Delays, Boeing Company.

[11] 31 January 2002, Air Traffic Management Working Together Team, Phase I System

Performance Requirements Document, D78010010-1.

[12] February 2001, Vision 2050: An Integrated National Transportation System, Federal Transportation Advisory Group.

[13] January 2001, European Aeronautics: A Vision for 2020, Meeting Society's Needs and Winning Global Leadership, European Commission for Research.

[14] 2002, Future Flight, Special Report 263, Transportation Research Board.
[15] Erzberger, H., December 2001, The Automated Airspace Concept, $4^{\text {th }}$ USA/Europe ATM R\&D Seminar.

[16] Duong, Vu, et al., December 2001, Sector-less Air Traffic Management, $4^{\text {th }}$ USA/Europe ATM R\&D Seminar.

[17] Aviation Traffic Activity Data System (ATADS), www.apo.data.faa.gov.

[18] 2001, 2000 Aviation Capacity Enhancement Plan, U.S. DOT/FAA.

[19] August 2002, Aviation Safety Statistical Handbook, DOT/FAA.

[20] 1997, NTSB Annual Review of Aircraft Accidents Data.

[21] March 2002 to October 2002, ATCSCC "F5" Miles-in-Trail (MIT) Restriction Logs. 